\title{
Neurologic complications of anesthesia
}

\section{A practical approach}

\author{
Alejandro A. Rabinstein, MD \\ Mark T. Keegan, MB, MRCPI, MSc, MD
}

\section{Summary}

Neurologic complications related to anesthesia are infrequent but can be serious. Neurologists are often consulted to evaluate patients with postoperative symptoms and must be ready to discriminate those truly caused by the anesthetic drug or procedure from the more common postoperative complications that are unrelated to the anesthesia itself. This practical review relies on cases to illustrate common reasons for neurologic consultation in the postsurgical setting. It also briefly summarizes what to expect when patients with central or peripheral neurologic disease undergo surgery under general or regional anesthesia.

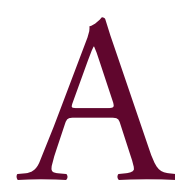

nesthesia has been unquestionably one of the greatest advances of modern medicine. It has allowed an enormous development of surgery with remarkable safety. Various anesthesia strategies are currently in use (general, regional, and local), and all of them are administered ev-

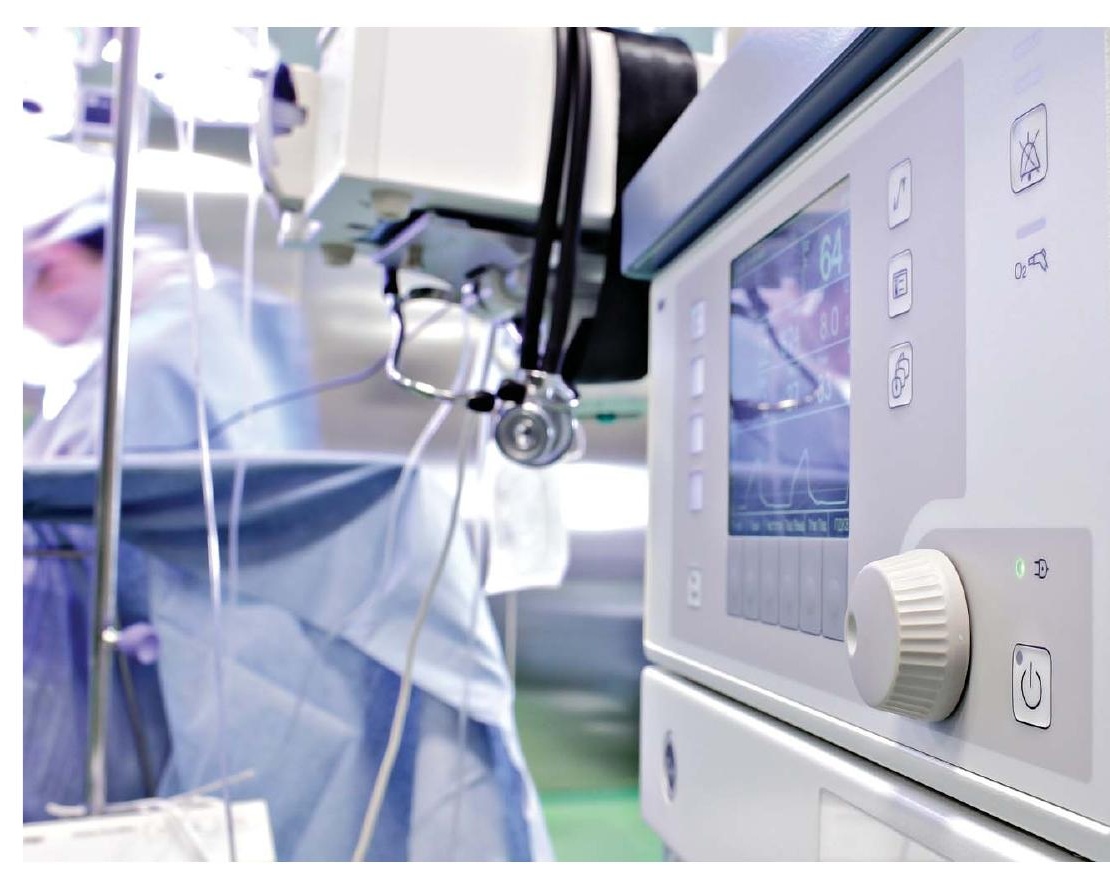
ery day around the world to large numbers of patients with extremely low rates of complications. Yet, among the infrequent complications that can occur in relation to anesthesia, those affecting the central and peripheral nervous system remain some of the most feared.

In this article we provide, with the help of illustrative vignettes, a brief overview of the most common neurologic complications related to anesthesia. We approach this broad and multifaceted topic with the consulting neurologist in mind. Thus, we have organized the content by the most common reasons for neurologic consultation and divided them into those related to general and regional anesthesia. Because of their clinical importance, we have included perioperative complications that are not caused by anesthesia, but may be mistakenly attributed to it. A brief section devoted to the discussion of the potential for neurologic complications in patients with established neurologic disorders is also included.

Departments of Neurology (AAR) and Anesthesia (MTK), Mayo Clinic, Rochester, MN.

Funding information and disclosures are provided at the end of the article. Full disclosure form information provided by the authors is available with the full text of this article at Neurology.org/cp.

Correspondence to: Rabinstein.alejandro@mayo.edu 


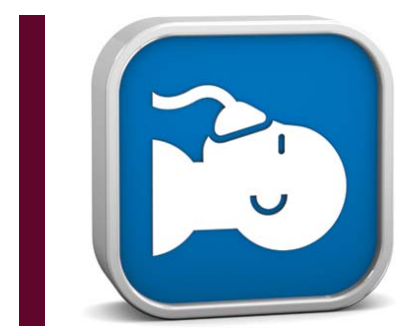

In patients with postoperative delirium, it is important to keep in mind the possibility of unexpected alcohol withdrawal because of its frequency and responsiveness to benzodiazepines.

\section{General anesthesia}

The patient is not waking up Vignette 1 A 55-year-old woman with no previous neurologic history undergoes an uncomplicated colectomy under general anesthesia for treatment of ulcerative colitis. Neurologic consultation is requested 6 hours after the conclusion of the surgery because the patient is still not awake enough to be extubated. In the surgical intensive care unit (ICU), the patient is stuporous but has normal brainstem reflexes (with very small, reactive pupils), conjugate gaze, normal muscle tone, symmetric localizing responses in both arms, withdrawal responses in both legs, and no adventitious movements. In addition to intraoperative opiate analgesia, she has received several doses of opiate since surgery given because of mild tachycardia deemed concerning for pain. Upon withholding opiates for the next 3 hours, the patient regains consciousness and is safely extubated.

Vignette 2 A 58-year-old man undergoes a complex and prolonged surgery for emergency treatment of a type A dissecting aortic aneurysm. He requires aggressive treatment for shock before and during the surgery. Postoperatively, he fails to arouse and Neurology is consulted the following day. Examination shows that the patient is stuporous and localizes with the right arm, but not the left. Head CT scan (figure 1) shows multifocal infarctions.

Vignette 3 A 68-year-old man is comatose after an ablation procedure for ventricular tachycardia complicated by refractory cardiogenic shock. Examination shows coma with bilaterally nonreactive, midposition pupils, preservation of corneal reflex only on one side, and no motor responses to pain. CT scan shows massive brain edema.

Failure to awaken after a surgery performed under general anesthesia is a common reason for urgent neurologic consultation in the hospital. ${ }^{1}$ Prolonged effects of anesthesia, particularly when combined with sedative effects of other medications (such as opiates) administered during and after surgery and in patients with liver or renal dysfunction, can explain some of these cases, as illustrated by vignette 1 . However, the main role of the consulting neurologist in these instances is to exclude primary neurologic disease. Stroke, global cerebral anoxia/ischemia, and status epilepticus need to be considered among the many other causes that can be responsible for the persistently impaired consciousness (table 1).

Careful history and neurologic examination are essential to reach the correct diagnosis and it is a frequent mistake to miss important information because the history is not readily available from an interview and the examination is deemed limited and confounded. Adequate history-taking in these cases should include a detailed review of the preoperative, intraoperative, and postoperative records as well as communication with the anesthesiologist and the surgeon. Adequacy of oxygenation, deviation from expected hemodynamic parameters during surgery, blood loss, and difficulties with hemostasis are pieces of information important to acquire. The neurologic examination should be focused on assessing the level of consciousness, brainstem reflexes, gaze, muscle tone, responses to pain, and presence of adventitious movements in the eyes, face, or limbs. Presence of lateralizing signs (e.g., asymmetric response to pain) demand consideration for brain imaging. Subtle nystagmus-like movements of the eyes or slight twitching in the face or fingers may be the only clinical expression of underlying status epilepticus. Rigidity and clonus should call attention to the possibility of drug toxicity (more on this later); asterixis can be the clue to hyperammonemia or other metabolic disturbances; myoclonus can be seen with various toxic and metabolic 
Figure 1 Perioperative brain infarctions

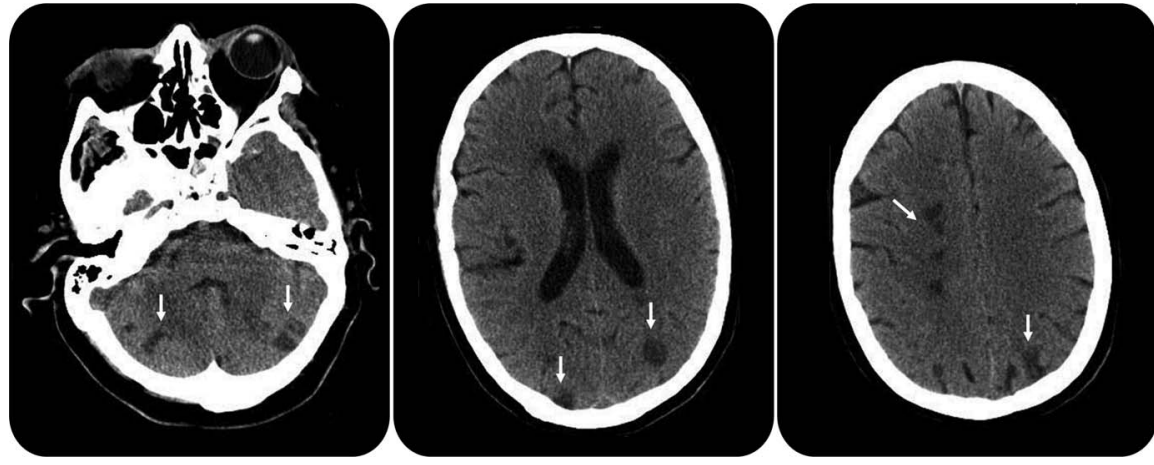

Noncontrast head CT scan of the patient described in vignette 2 shows multiple embolic infarctions (arrows).

encephalopathies, but also after severe brain anoxia. The value of a detailed general physical examination should not be forgotten. For instance, in the very early postoperative phase, close attention to the depth and pattern of respiration is crucial and should alert us to the possibility of $\mathrm{CO}_{2}$ narcosis. Meanwhile, splinter hemorrhages in the conjunctivas or nail beds can be the clue to the diagnosis of fat embolism in patients who fail to arouse after the repair of a long bone fracture.

Additional testing should also be focused and directed by the findings on physical examination. Many patients just need more time and waiting before ordering tests is often the right decisionand the most cost-effective-when careful history and physical examination have not raised red flags. Brain imaging is indicated in patients with new focal deficits. While a CT scan can be useful to detect hemorrhages, territorial ischemic infarctions, and massive brain edema, an MRI may be necessary to diagnose embolic showers and more subtle manifestations of global brain anoxia/ischemia (figure 2). EEG should be reserved for patients with suspected seizures and in those cases continuous monitoring may be more informative. Lumbar puncture is rarely necessary in patients with postoperative encephalopathy. Selective use of blood tests is recommended; serum creatinine and blood urea to exclude renal failure, serum ammonia in cases of suspected liver insufficiency, and neuronal specific enolase in patients with suspected anoxic brain damage should be considered for diagnostic or prognostic purposes.

The patient is confused and agitated Vignette 4 A 78-year-old woman is very agitated in the surgical ICU after undergoing spine surgery under general anesthesia the day before. She was taking multiple psychotropic medications before the surgery including a benzodiazepine for anxiety and opiates for chronic pain. Her agitation becomes controlled with dexmedetomidine, which allows extubation, and improves progressively over the following days, with gradual and selective resumption of her outpatient medications.

Postoperative delirium is a very frequent complication, especially in elderly patients, patients with preexistent cognitive impairment, and those receiving polypharmacy (especially if including benzodiazepines) before surgery. ${ }^{2,3}$ Reported incidences vary across studies, but may exceed $50 \%$ in high-risk patients. There is very scant evidence regarding the influence of the type of anesthesia on the risk of postoperative delirium. In elderly patients undergoing surgery for hip fracture repair under spinal anesthesia, keeping a lighter degree of intraoperative sedation using propofol (as compared to deep sedation) has been associated with reduction in the rate of postoperative delirium. ${ }^{4}$ Postoperative use of benzodiazepines increases the risk of delirium, and therefore they should be avoided. Dexmedetomidine is a better option when sedation is needed. For agitation, antidopaminergic drugs, such as haloperidol, quetiapine, or olanzapine, should be the first choice. Physostigmine may be useful in the postanesthesia care unit for the treatment of delirium associated with the administration of centrally acting anticholinergic agents. Cholinergic enhancement with donepezil has been proposed to decrease the risk of 


\section{Table 1 Principal causes of postoperative impairment of consciousness}

Prolonged anesthetic and analgesic effect

Advanced age

Decreased metabolism and clearance

Toxic encephalopathy

Perioperative drugs (benzodiazepines, opiates, serotonergic agents)

Drug interactions

Metabolic encephalopathy

Renal failure

Liver failure

Hypercapnia and hypoxia

Hypoglycemia

Hyponatremia and other electrolyte abnormalities

Hyperosmolality

Acidemia

Septic encephalopathy

Acute stroke

Ischemic

Thromboembolism

Fat embolism

Air embolism

Hemodynamic hypoperfusion

Hemorrhagic

Seizures and status epilepticus (convulsive and nonconvulsive)

Anoxic/ischemic global brain injury

delirium after hip surgery in elderly patients, but results of small pilot studies have been disappointing. ${ }^{5,6}$ In patients with postoperative delirium, it is important to keep in mind the possibility of unexpected alcohol withdrawal because of its frequency and responsiveness to benzodiazepines.

It is important to remember that most cases of postoperative delirium are hypoactive. The misconception to equate delirium with agitation is very common and conspires against prompt recognition and management of delirium when it does not present with hyperactive signs. Routine use of standardized and validated scores for assessment of level of sedation (like the Richmond Agitation-Sedation scale) and screening for delirium (like the CAM-ICU) is highly advisable in the postoperative period (figure 3 ).

The patient is rigid and febrile Vignette 5 A 57-year-old woman with major depressive disorder on multiple psychotropic medications has emergency abdominal surgery under general anesthesia for gallbladder abscess. After surgery she remains markedly febrile and is noted to be rigid. On examination, she is barely arousable, but when aroused becomes agitated. She is diaphoretic, tachycardic, and has diffuse rigidity (more severe in the legs), tremors, and sustained clonus. Her medications include clomipramine, duloxetine, trifluoperazine, benztropine, fentanyl, and oxycodone. Discontinuation of serotonin-enhancing medications, combined with administration of cyproheptadine and brief infusion of dexmedetomidine to control the periods of agitation and to ameliorate the tachycardia, is followed by gradual resolution of all neurologic symptoms. 
Figure 2 Value of MRI of the brain in patients with altered consciousness after surgery

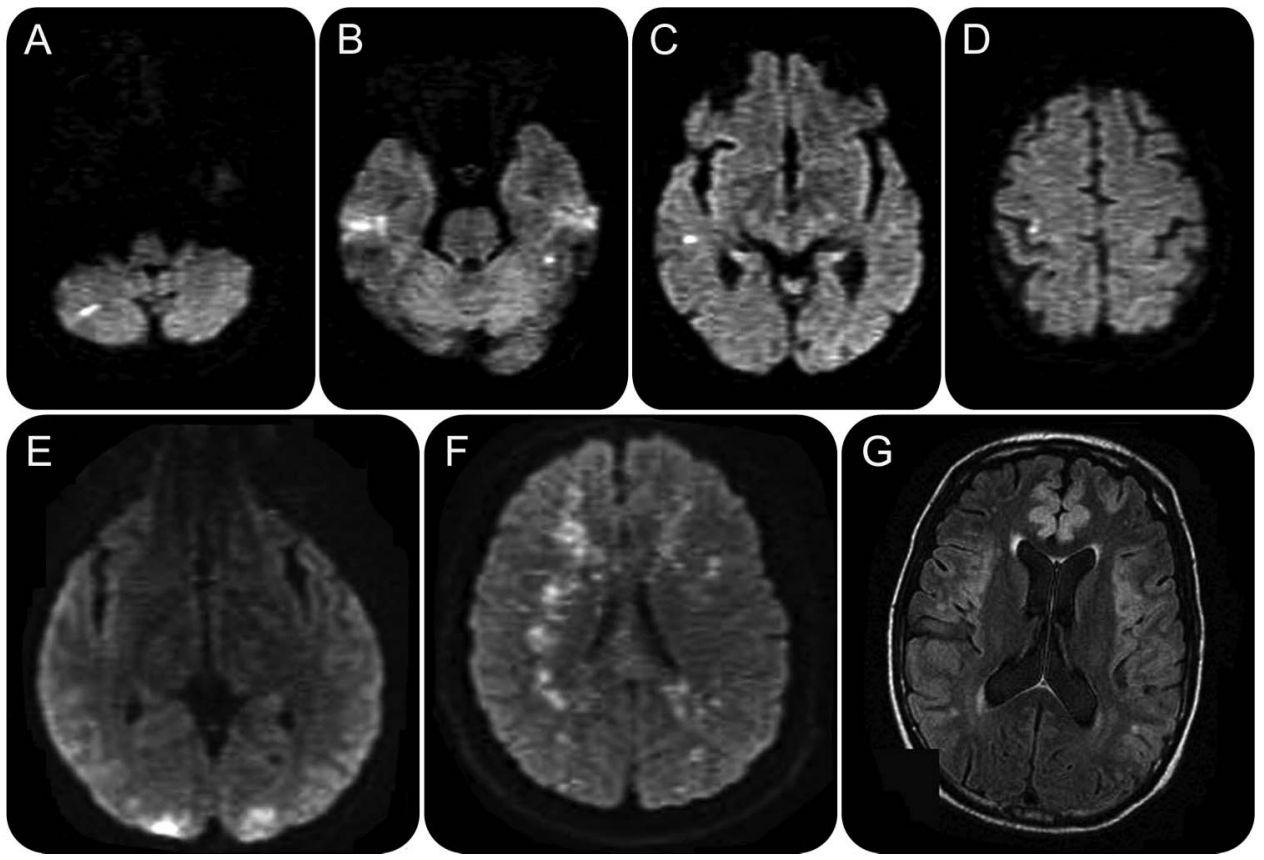

(A-D) Diffusion-weighted imaging (DWI) sequence of a patient with multiple small infarctions caused by an embolic shower, which could not be visualized on CT scan. (E) DWI of a patient with top-of-the-basilar syndrome diagnosed after a cardiovascular procedure. He recovered consciousness but had persistent bilateral visual loss from bilateral occipital infarctions. (F) DWI of a patient with fat embolism after repair of a complex long bone fracture shows the typical "star-field appearance." (G) Fluid-attenuated inversion recovery sequence of a patient with severe cortical laminar necrosis after an emergency vascular surgery preceded by profound hypoxia and shock and complicated by refractory intraoperative hypotension.

When evaluating a febrile and rigid patient, the differential diagnosis encompasses serotonin syndrome, neuroleptic malignant syndrome, and malignant hyperthermia. Serotonin syndrome is the most commonly seen in the postoperative period. Patients at risk are those taking drugs with serotonergic effects. The list of these medications is long and includes antidepressants, mood stabilizers, antihistamines, triptans, muscle relaxants, and amphetamines, but also opiates, tramadol, and antiemetics (ondansetron, metoclopramide) often used after surgery. ${ }^{7}$ Drug interactions are typically responsible for the emergence of the syndrome. The most characteristic clinical features are those presented in vignette 5. Patients also have reactive mydriasis, diffuse hyperreflexia, and hyperactive bowel sounds. The predominance of rigidity in the lower over the upper limbs is quite distinctive of this syndrome and useful in practice. Cyproheptadine (4-8 mg every 6 hours) may accelerate recovery, but the most important prescription should be the immediate discontinuation of all serotonergic drugs.

Neuroleptic malignant syndrome is often suspected, but much less common. Distinguishing features are exposure to antidopaminergic drugs, less acute presentation (over 1-3 days rather than hours for serotonin syndrome), bradykinesia, lead-pipe rigidity equally involving all limbs, hyporeflexia, and normal/diminished bowel sounds. Before depression of consciousness occurs, patients with neuroleptic malignant syndrome are progressively more hypokinetic, while patients with serotonin syndrome have excessive movements. Signs of autonomic instability (e.g., fever, blood pressure lability, diaphoresis) are typically present. Creatine phosphokinase levels are often elevated. When the diagnosis of neuroleptic malignant syndrome is considered, antidopaminergic medications should be stopped without delay and patients should be carefully monitored for the possibility of complications from dysautonomia and excessive muscle rigidity. Dopamine agonists, such as bromocriptine, can help accelerate the recovery. Dantrolene, a potent muscle relaxant, is useful in patients with very severe rigidity. 
Figure 3 Sedation and delirium assessment tools

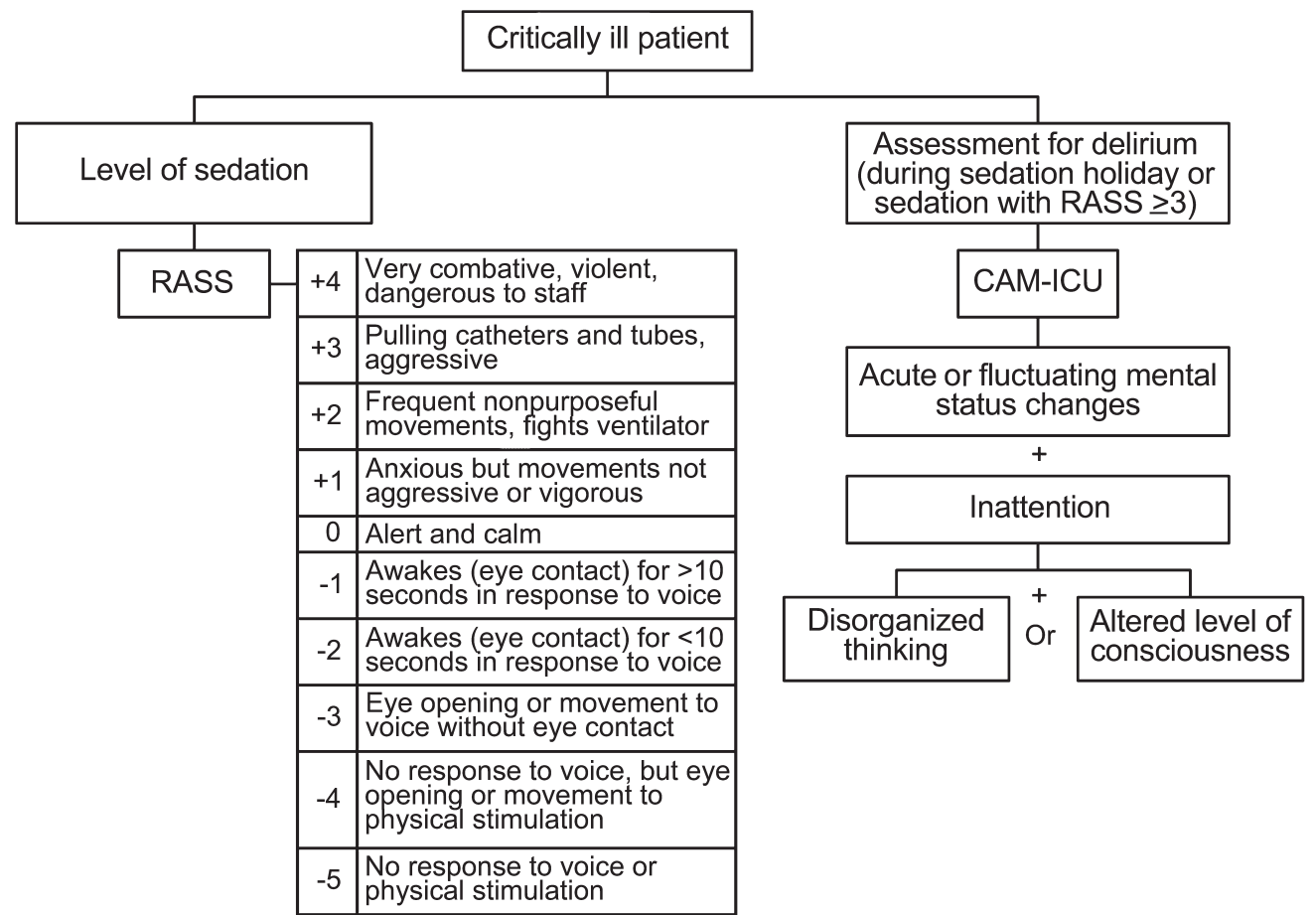

Evaluation of level of sedation and delirium using the Richmond Agitation Sedation Scale (RASS) and Confusion Assessment Method for the Intensive Care Unit (CAM-ICU).

Malignant hyperthermia is very rarely seen by neurologists because it occurs in the operating room upon anesthesia induction and neuromuscular paralysis or within minutes of anesthesia cessation. ${ }^{8}$ This genetic disorder is caused by mutations affecting receptors that control the transport of calcium from the sarcoplasmic reticulum to the cytoplasm. Exposure to inhalational anesthetics, especially when succinylcholine is administered, results in excessive availability of calcium in the cytoplasm, which in turn provokes sustained muscle contraction. A hypermetabolic crisis follows, manifested by hypercapnia, hyperthermia, and mixed acidosis. Severe rhabdomyolysis and cardiac arrhythmias can occur if diagnosis is delayed. Immediate discontinuation of the inhalational agent and administration of dantrolene are extremely effective in reversing the syndrome.

The patient is seizing Vignette 6 A 38-year-old alcoholic man with a previous traumatic brain contusion falls down 2 flights of stairs and sustains an exposed leg fracture. Following surgical treatment under general anesthesia, he remains tremulous and restless. He then has a generalized convulsion.

Apart from neurosurgeries, surgical intervention is not a predisposing factor for seizures. Most anesthetics (isoflurane, desflurane, propofol, benzodiazepines) have antiepileptic properties. Seizures may occur following anesthesia, but they are very uncommon. ${ }^{9}$ Risk factors include previous epilepsy (especially among children), previous structural brain injury, and substance abuse. When postoperative seizures occur in patients with epilepsy, they are generally related to poor preoperative control of the epilepsy and not to the anesthesia type. ${ }^{10}$ Yet in patients with epilepsy it is prudent to avoid drugs known to induce epileptiform changes, such as sevoflurane, etomidate, lidocaine, and short-acting opiates (remifentanil, sufentanil). ${ }^{11}$ Epileptic seizures should be differentiated from seizure-like movements (most notably myoclonus often seen upon propofol withdrawal) and nonepileptic behavioral seizures. When a patient with postoperative seizures fails to regain consciousness, it is always prudent to pursue continuous EEG monitoring to exclude nonconvulsive status epilepticus. 


\section{Patients with Parkinson disease can have transient worsening lespecially if antiparkinsonian medications are stopped for too long) and delirium after surgery, but generally no lasting decline.}

The patient cannot see Vignette 7 Following a complex spinal fusion, a 55-year-old obese man complains of bilateral visual loss. On examination, the patient is nearly blind and funduscopy is unremarkable.

Posterior ischemic optic neuropathy is an exceptional but potentially devastating complication of spinal fusion surgery. The usual scenario is a difficult surgery requiring prolonged prone positioning, associated with large blood loss in an obese man. The performance of staged surgeries, a more liberal intraoperative transfusion strategy, avoidance of intraoperative hypotension, and maintenance of the head at or above the level of the heart have been recommended, but the etiology of the condition is unclear and the value of these strategies is unproven. ${ }^{12}$ Although the visual impairment can be transient, it is often severe and bilateral. Cardiac surgeries can also be rarely complicated by visual loss, typically due to anterior ischemic optic neuropathy or retinal embolism. ${ }^{13}$ The anesthesia technique does not appear to be associated with the risk of perioperative visual loss. ${ }^{12,13}$ Cortical blindness can be a manifestation of posterior reversible encephalopathy syndrome in patients who have marked blood pressure fluctuations during or after surgery.

\section{Regional anesthesia}

Regional anesthesia techniques include neuroaxial blockade, either spinal (intrathecal) or epidural (extrathecal), and peripheral nerve block (most commonly targeting the brachial plexus, lumbosacral plexus, or their components). They offer an excellent safety profile, but various neurologic complications can rarely occur (table 2).

The patient cannot move his or her legs Vignette 8 A 79-year-old man with atrial fibrillation on oral anticoagulation undergoes a hip replacement under regional anesthesia (epidural block). International normalized ratio (INR) the day before surgery is 1.7. Postoperatively, the epidural catheter is kept in place for analgesia. That night he is moving his legs well. The next morning, within a couple of hours of removal of the epidural catheter, he develops bilateral leg numbness and then becomes paraparetic. Examination confirms paraplegia with anesthesia in both legs to all sensory modalities below T12 level. MRI scan reveals a low thoracic epidural hematoma. After emergent surgical evacuation, the patient regains partial leg function.

Epidural hematoma may be the most feared complication of spinal and epidural blockade, but it is very uncommon. In fact, the vignette is constructed based on reported cases in the literature rather than our personal experience. Estimated incidence is lower than $1 / 15,000$ for epidural and lower than 1/22,000 spinal anesthesia cases, but the risk may be higher with older age, vertebral column skeletal abnormalities, difficulty placing the needle, coagulopathy, and the presence of an indwelling epidural catheter during anticoagulation. ${ }^{14}$ The American Society of Regional Anesthesia and Pain Medicine has published a consensus statement with recommendations to minimize the risk of epidural hematoma related to regional anesthesia in patients receiving antithrombotics or fibrinolytics. ${ }^{14}$

As illustrated by vignette 8 , the presentation of epidural hematoma is often more gradual than that of spinal cord infarction. Compressive symptoms may be initially sensory and then motor. The hematoma may form upon placement or withdrawal of the epidural catheter. MRI is the preferred diagnostic modality. Emergent surgical evacuation can reverse the deficits, but outcomes vary depending on the duration and severity of the compression. ${ }^{15}$ 


\section{Table 2 Main neurologic complications from regional anesthesia}

\section{Neuropathy}

Epidural hematoma

Epidural abscess

Orthostatic headaches (after dural puncture)

Aseptic meningitis

Signs of central toxicitya

Worsening or relapse of previous neurologic disease (especially neuropathy)

Seizures

Accidental total spinal anesthesiab

aDizziness, lightheadedness, perioral numbness, adventitious movements, visual and auditory disturbances.

b When an intended epidural anesthetic injection is unintentionally administered into the subarachnoid space, patients can develop unconsciousness with marked bilateral mydriasis, quadriplegia, and cardiorespiratory collapse.

Vignette 9 A 48-year-old woman has an uncomplicated vaginal hysterectomy under spinal anesthesia. The following day, the neurologist is consulted because the patient is having pain in the buttocks and both legs, and some difficulty moving her legs.

Regional anesthesia is very safe. In a study of almost 160,000 blocks, the incidence of serious complications (seizure, central or peripheral neural injury, death) was 3.5 per 10,000 blocks. ${ }^{16}$ The risk of neuropathy associated with regional anesthesia is very low. The mechanisms of nerve damage include mechanical trauma (direct injury caused by the needle or the catheter or by intraneural injection, or indirect injury due to a compressing hematoma), local anesthetic toxicity (the risk of which increases with the local anesthetic dose and concentration), and epinephrine use (either by a direct ischemic mechanism or an indirect pharmacokinetic effect on local anesthetic clearance). The term transient neurologic syndrome is used in the anesthesia literature to refer to temporary pain or dysesthesia in the legs or buttocks following spinal anesthesia, especially when lidocaine is used and the surgery is performed in the lithotomy position. ${ }^{17}$

When all perioperative neuropathies are considered, the nerves most commonly affected are the ulnar, common peroneal, femoral, and sciatic nerves, as well as the brachial and lumbosacral plexi. ${ }^{18}$ Regardless of the type of anesthesia used, intraoperative positioning predisposing to stretching or compression is deemed a common cause. Manifestations are usually sensory or sensorimotor. The risk is higher in men, smokers, obese, and very thin patients, and those with diabetes and hypertension. ${ }^{19}$ Most of these perioperative neuropathies are transient and full recovery is the most frequent outcome in our experience.

The patient has a terrible headache Vignette 10 After an uncomplicated orthopedic surgery under spinal anesthesia, a previously healthy 34-year-old woman has severe headaches that keep her in bed.

Dural puncture can cause a persistent leak of CSF and the ensuing intracranial hypotension manifests with orthostatic headaches (i.e., worsened when patient is upright and ameliorated by recumbency). This complication is more common in young women. Cases provoked by spinal anesthesia (and accidental dural puncture when performing epidural anesthesia) can cause severe headache but generally have minimal long-term implications and respond well to epidural blood patch. In contrast, CSF leaks generated during complex spine surgeries can produce more disabling symptoms, lead to additional complications (e.g., depressed consciousness, brain sagging, subdural hemorrhages), and require additional surgery for repair of the dural tear. 


\section{Anesthesia in patients with neurologic disease}

A common question asked by patients, families, and colleagues is whether a patient with a neurologic disease affecting the central or peripheral nervous systems might get worse because of exposure to anesthesia during surgery. The short answer is no in most cases, regardless of whether the anesthesia is general or regional. ${ }^{20}$ However, there are some nuances that deserve attention. There is no conclusive clinical evidence that anesthetics of any type can cause neurodegeneration, but preoperative cognitive impairment and dementia are associated with increased risk of postsurgical delirium, cognitive decline, and poor functional recovery. ${ }^{21}$ The anesthetic type and regimen used does not appear to affect these risks. Patients with Parkinson disease can have transient worsening (especially if antiparkinsonian medications are stopped for too long) and delirium after surgery, but generally no lasting decline. The presence of preoperative dysautonomia (e.g., from multiple system atrophy) should be noted to the anesthesiologist before surgery. Monitored anesthesia care should be considered in patients with amyotrophic lateral sclerosis, because weaning from ventilation may be very challenging; yet general anesthesia (ideally without paralytic agents) can be applied safely when necessary. Issues related to general anesthesia in epileptic patients were briefly discussed before; regional blockade can also be administered safely to these patients. Although there have been isolated case reports of multiple sclerosis worsening after surgery, these occurrences are exceptional. Presurgical planning in myasthenic patients might need to include treatment with plasma exchange to reduce the risk of exacerbation after surgery. Patients with severe peripheral neuropathy undergoing neuroaxial anesthesia can have new or worsened postoperative symptoms, but the reported risk is very low $(<0.5 \%)^{22}$

\section{REFERENCES}

1. Anastasian ZH, Ornstein E, Heyer EJ. Delayed arousal. Anesthesiol Clin 2009;27:429-450.

2. McPherson JA, Wagner CE, Boehm LM, et al. Delirium in the cardiovascular ICU: exploring modifiable risk factors. Crit Care Med 2013;41:405-413.

3. Lee HB, Mears SC, Rosenberg PB, et al. Predisposing factors for postoperative delirium after hip fracture repair in individuals with and without dementia. J Am Geriatr Soc 2011;59:2306-2313.

4. Sieber FE, Zakriya KJ, Gottschalk A, et al. Sedation depth during spinal anesthesia and the development of postoperative delirium in elderly patients undergoing hip fracture repair. Mayo Clin Proc 2010;85:18-26.

5. Marcantonio ER, Palihnich K, Appleton P, Davis RB. Pilot randomized trial of donepezil hydrochloride for delirium after hip fracture. J Am Geriatr Soc 2011;59(suppl 2):S282-S288.

6. Sampson EL, Raven PR, Ndhlovu PN, et al. A randomized, double-blind, placebo-controlled trial of donepezil hydrochloride (Aricept) for reducing the incidence of postoperative delirium after elective total hip replacement. Int J Geriatr Psychiatry 2007;22:343-349.

7. Boyer EW, Shannon M. The serotonin syndrome. N Engl J Med 2005;352:1112-1120.

8. Denborough M. Malignant hyperthermia. Lancet 1998;352:1131-1136.

9. Benish SM, Cascino GD, Warner ME, et al. Effect of general anesthesia in patients with epilepsy: a population-based study. Epilepsy Behav 2010;17:87-89.

10. Niesen AD, Jacob AK, Aho LE, et al. Perioperative seizures in patients with a history of a seizure disorder. Anesth Analg 2010;111:729-735.

11. Voss LJ, Sleigh JW, Barnard JP, Kirsch HE. The howling cortex: seizures and general anesthetic drugs. Anesth Analg 2008;107:1689-1703.

12. Postoperative Visual Loss Study Group. Risk factors associated with ischemic optic neuropathy after spinal fusion surgery. Anesthesiology 2012;116:15-24.

13. Roth S. Perioperative visual loss: what do we know, what can we do? Br J Anaesth 2009;103(suppl 1): i31-i40.

14. Horlocker TT, Wedel DJ, Rowlingson JC, et al. Regional anesthesia in the patient receiving antithrombotic or thrombolytic therapy: American Society of Regional Anesthesia and Pain Medicine Evidence-Based Guidelines (Third Edition). Reg Anesth Pain Med 2010;35:64-101.

15. Vandermeulen EP, Van Aken H, Vermylen J. Anticoagulants and spinal-epidural anesthesia. Anesth Analg 1994;79:1165-1177.

16. Auroy Y, Benhamou D, Bargues L, et al. Major complications of regional anesthesia in France: the SOS regional anesthesia hotline service. Anesthesiology 2002;97:1274-1280. 
17. Zaric D, Pace NL. Transient neurologic symptoms (TNS) following spinal anaesthesia with lidocaine versus other local anaesthetics. Cochrane Database Syst Rev 2009;CD003006.

18. Warner MA. Perioperative neuropathies. Mayo Clin Proc 1998;73:567-574.

19. Welch MB, Brummett CM, Welch TD, et al. Perioperative peripheral nerve injuries: a retrospective study of 380,680 cases during a 10-year period at a single institution. Anesthesiology 2009;111:490-497.

20. Rabinstein AA. Neurologic complications of anesthesia. Continuum 2011;17:134-147.

21. Robinson TN, Raeburn CD, Tran ZV, et al. Postoperative delirium in the elderly: risk factors and outcomes. Ann Surg 2009;249:173-178.

22. Hebl JR, Kopp SL, Schroeder DR, Horlocker TT. Neurologic complications after anesthesia or analgesia in patients with preexistent peripheral sensorimotor neuropathy or diabetic polyneuropathy. Anesth Analg 2006;103:1294-1299.

\section{STUDY FUNDING}

No targeted funding reported.

\section{DISCLOSURES}

A. Rabinstein serves on a safety monitoring board for ALIAS and PREVAIL trials; serves on the editorial board of Neurology ${ }^{\circledast}$ and as an Associate Editor of Neurocritical Care; receives publishing royalties for Practical Neuroimaging in Stroke (Elsevier, 2009) and What To Do? Neurocritical Care (Oxford, 2011); and receives research support from CardioNet and DJO Global. M.T. Keegan has received speaker honoraria from Dannemiller and serves on the editorial boards of Mayo Clinic Proceedings Critical Care Research and Practice ASA ACE. Full disclosure form information provided by the authors is available with the full text of this article at Neurology.org/cp.

\section{Related articles from other AAN physician and patient resources}

\section{Continuum: Lifelong Learning in Neurology ${ }^{\circledR} \quad$ - www.ContinuumJournal.com}

Neurologic complications of anesthesia

February 2011;134-147.

\section{Neurology Now ${ }^{\circledR} \quad \bullet \quad$ www.neurologynow.com}

Dealing with delirium: New research suggests that hospital delirium can be more than just a temporary inconvenience

December/January 2012;8:33-35.

$$
\text { Neurology Today }{ }^{\circledR} \quad \bullet \quad \text { www.neurotodayonline.com }
$$

Concerns remain after FDA meeting on neurotoxic effects of pediatric anesthesia May 15, 2007;7:24-25. 